\title{
AVALIAÇÃO DO CONSUMO DE ÁLCOOL ENTRE GESTANTES CADASTRADAS NO SISPRENATAL EM LONDRINA/PR*
}

\author{
Pollyana Bortholazzi Gouvea ${ }^{1}$, Sarah Nancy Deggau Hegeto de Souza², Maria do Carmo Lourenço Haddad ${ }^{3}$, Débora
} Falleiros de Mello ${ }^{4}$

\begin{abstract}
RESUMO: Este estudo teve como objetivos investigar o consumo de álcool entre gestantes cadastradas no SisPreNatal em regiões da cidade de Londrina, identificar padrões de consumo de álcool e relacionar fatores sociodemográficos com o consumo de álcool. Foram entrevistadas 202 gestantes, de junho a setembro de 2007, utilizando-se o instrumento de rastreamento para o uso nocivo de álcool (T-ACE). Entre as participantes, 19,3\% foram T-ACE positivo no primeiro trimestre; 3,9\% afirmaram consumir mais de $56 \mathrm{~g}$ de álcool $/ \mathrm{mês}$ e 2,5\% afirmaram consumir mais de $29 \mathrm{~g}$ álcool $/ \mathrm{semana}$. A partir do $2^{\circ}$ trimestre, a maioria referiu fazer pouco consumo de bebidas alcoólicas e muitas afirmaram não fazer mais uso após saber da gravidez. A maioria era pertencente à classe média baixa. O estudo demonstrou que uma quantidade significativa de mulheres consumiu bebida alcoólica pelo menos por um período da gestação, oferecendo riscos ao feto. PALAVRAS-CHAVE: Alcoolismo; Gravidez; Síndrome alcoólica fetal.
\end{abstract}

\section{ASSESSMENT OF ALCOHOL CONSUMPTION AMONG PREGNANT WOMEN REGISTERED IN SISPRENATAL IN LONDRINA / PR}

\begin{abstract}
This study aimed to investigate alcohol consumption among pregnant women enrolled in SisPreNatal in areas of the city of Londrina, and identify patterns of alcohol consumption and sociodemographic factors related to the consumption of alcohol. We interviewed 202 pregnant women, from June to September 2007 using the screening instrument for harmful use of alcohol (T-ACE). Among the participants, 19.3\% were T-ACE positive in the first three months of pregnancy, $3.9 \%$ reported consuming more than $56 \mathrm{~g}$ of alcohol per month and $2.5 \%$ reported consuming more than $29 \mathrm{~g}$ alcohol per week. From the second trimester, most of the pregnant women reported little consumption of alcoholic beverages and many said they make no further use after knowing they were pregnant. Most belonged to lower or middle social class. The study showed that a significant number of women consumed alcohol at least for a period during pregnancy, posing risks to the fetus.
\end{abstract}

KEYWORDS: Alcoholism; Pregnancy; Fetal alcohol syndrome.

\section{EVALUACIÓN DEL CONSUMO DE ALCOHOL ENTRE GESTANTES DEL CATASTRO DE SISPRENATAL EN LONDRINA/PR}

RESUMEN: Este estudio tuvo el objetivo de investigar el consumo de alcohol entre gestantes del catastro de SisPreNatal en regiones de la ciudad de Londrina, identificar patrones de consumo de alcohol y relacionar fatores sociodemográficos con consumo de alcohol. Fueron entrevistadas 202 gestantes, de junio a septiembre de 2007, utilizándose el instrunento que rastrea EL uso nocivo de alcohol (T-ACE). Entre las participantes, 19,3\% fueron T-ACE positivo en primero trimestre; $3,9 \%$ afirmaron consumir más de $56 \mathrm{~g}$ de alcohol/mês y $2,5 \%$ afirmaron consumir más de $29 \mathrm{~g}$ alcohol $/ \mathrm{semana}$. A partir del $2^{\circ}$ trimestre, la mayoría declaró hacer poco consumo de bebidas alcohólicas y muchas dicieron no hacer más uso después de la certidumbre de la gravidez. La mayoria pertenenía a la clase media baja. El estudio ha demostrado que una cuantidade significativa de mujeres consumió bebida alcohólica por lo menos por un periodo de la gestación, ofreciendo riesgos al feto.

PALABRAS CLAVE: Alcoholismo; Gravidez; Síndrome alcohólica fetal.

\footnotetext{
*Artigo extraído de monografia de conclusão de Curso de Graduação em Enfermagem pela Universidade Estadual de Londrina-UEL. ${ }^{1}$ Enfermeira. Mestranda pelo Programa de Pós-Graduação em Enfermagem Fundamental da Universidade de São Paulo-USP.

${ }^{2}$ Enfermeira. Doutoranda em Enfermagem pelo Programa de Pós-Graduação da USP. Docente do Departamento de Enfermagem da UEL. ${ }^{3}$ Enfermeira. Doutor em Enfermagem. Docente do Curso de Enfermagem da UEL.

${ }^{4}$ Enfermeira. Doutor em Enfermagem. Docente do Curso de Enfermagem da Escola de Enfermagem de Ribeirão Preto-EERP/USP.
}

Autor correspondente:

Pollyana Bortholazzi Gouvea

Universidade Estadual de Londrina

R. Ernani Lacerda de Athayde, 210 - 86055-630 - Londrina-PR-Brasil

Recebido: 19/04/10

E-mail: pollyana_bortholazzi@yahoo.com.br Aprovado: 19/08/10 


\section{INTRODUÇÃO}

O hábito de beber é um costume muito antigo que acompanha a humanidade desde seus primórdios e esteve presente em todas as culturas. Porém, a partir da Revolução Industrial se formaram as grandes concentrações urbanas e o acesso ao álcool tornou-se mais fácil, fazendo com que esta substância, cheia de significados, também se transformasse em um problema de saúde pública. Assim, o alcoolismo tornou-se um importante problema social, com causalidade multifatorial, não sendo possível diferenciar as influências genéticas das ambientais ${ }^{(1)}$.

Nas mulheres, houve um aumento considerável do uso desta substância, principalmente após a ampliação do seu espaço social, junto ao aumento da disputa pelo mercado de trabalho( ${ }^{(2)}$. Acrescentase ainda que, devido aos altos níveis de ansiedade presente em muitas mulheres, a ingesta de etílicos é incentivada, visando reduzir estados de tensão e preocupação, embora o consumo crônico de álcool possa desencadear estados ansiosos ${ }^{(3)}$.

Durante a gestação, o consumo de bebidas alcoólicas envolve um risco ainda maior, já que existe possibilidade de ocorrer teratogenicidade e embriotoxicidade; e, como agravante, muitas mulheres fazem uso de álcool sem saberem que estão grávidas. Podese também levar em consideração que nem sempre o álcool causará problemas ao feto, porém, estudos têm demonstrado a influência negativa exercida pelo álcool quando ingerido na gestação ${ }^{(4)}$.

Depois de ser ingerido pela gestante, o álcool atravessa a barreira placentária fazendo com que o feto atinja os mesmos níveis de concentração alcoólica que a mãe. Devido à maior lentidão do metabolismo e eliminação fetal, o líquido amniótico fica impregnado de álcool não modificado (etanol) e acetaldeído (metabólito do etanol), ocasionando um tempo de exposição maior do feto ${ }^{(5)}$.

Para o feto, o uso de álcool durante a gravidez resulta em uma série de consequências conhecidas como desordens alcoólicas fetais; seus efeitos teratogênicos estão relacionados com o nível de exposição fetal ao mesmo. Dentre as complicações estão presentes anomalias físicas, dismorfismo, aumento da incidência de abortamento espontâneo, descolamento prematuro de placenta, hipertonia uterina, trabalho de parto prematuro e líquido amniótico meconial $^{(6-7)}$.

A incidência de anomalias congênitas em filhos de mulheres bebedoras foi estimada em cerca de $32 \%$ e em $12 \%$ para todas as mulheres grávidas (alcoólatras ou não), se beberem durante o primeiro trimestre de gestação ${ }^{(8)}$.

A Síndrome Alcoólica Fetal (SAF) é uma dessas desordens e representa o final grave de uma gama de efeitos, inclusive a morte fetal. Sua etiologia tem sido atribuída ao potencial mutagênico do acetaldeído, à capacidade do etanol para alterar a membrana celular e interferir na síntese de proteínas, e às alterações nos níveis de prostaglandinas, promovendo uma vasoconstrição e favorecendo, assim, um quadro de hipóxia crônica no ambiente uterino ${ }^{(9)}$.

A SAF é uma condição permanente e afeta todos os aspectos da vida do indivíduo e de sua família. É identificada por características faciais anormais, deficiência do crescimento e problemas no Sistema Nervoso Central (SNC). Pessoas com história de SAF podem ter problemas com aprendizado, memória, atenção, comunicação, visão, audição ou uma combinação destes, que podem conduzir a dificuldades na escola e nos relacionamentos ${ }^{(9)}$.

$\mathrm{O}$ rastreamento e intervenção em mulheres em risco de exposição ao álcool durante a gestação pode prevenir a SAF e outras desordens relacionadas ao álcool ${ }^{(10)}$.

Embora a confirmação do uso de bebidas alcoólicas pelas gestantes não ser um assunto de fácil abordagem, principalmente pelos estigmas sociais que existem, além de causar constrangimento das mesmas em informar a respeito, questionou-se qual seria a atual situação do consumo de álcool entre as gestantes cadastradas no SisPreNatal, em Unidades Básicas de Saúde de Londrina-PR.

Portanto, o presente estudo tem como objetivo investigar o consumo de álcool entre as gestantes cadastradas no SisPreNatal em Londrina/PR, identificar padrões de consumo de álcool em uma população de gestantes por meio de instrumentos validados de rastreamento de uso de álcool e relacionar fatores sociodemográficos com o consumo de álcool.

\section{METODOLOGIA}

O estudo foi realizado de junho a outubro de 2007, em Londrina, Estado do Paraná, que dispõe de 52 Unidades Básicas de Saúde (UBS), as quais contam com, aproximadamente, 90 equipes de Saúde da Família onde atuam médicos, enfermeiros, auxiliares de enfermagem e agentes comunitários de saúde. 
O município possui uma rede de acompanhamento de pré-natal à gestantes cadastradas no SisPreNatal, que é um sistema criado pelo DATASUS, e que tem por objetivo o desenvolvimento de Ações de Promoção, Prevenção e Assistência à Saúde de Gestantes e Recém-Nascidos. Ele visa reduzir as altas taxas de morbimortalidade materna, perinatal e neonatal, melhorando o acesso da cobertura e qualidade do acompanhamento pré-natal, da assistência ao parto e puerpério e da assistência neonatal ${ }^{(11)}$.

Este sistema também fornece informações fundamentais para o planejamento, acompanhamento e avaliação das ações desenvolvidas, por meio do Programa de Humanização ao Pré-Natal e Nascimento, ou seja, no SisPreNatal está definido o elenco mínimo de procedimentos para uma assistência pré-natal adequada $^{(11)}$.

Primeiramente, por meio de ligações telefônicas, foi feito um levantamento aproximado do total de gestantes cadastradas no SisPreNatal em todas UBS, sendo identificado o número de gestantes cadastradas em cada uma das Unidades. Constatou-se que, até junho de 2007, haviam 2.231 gestantes cadastradas.

Após o levantamento desses dados, foram selecionadas cinco Unidades Básicas de Saúde, uma por região, de acordo com o número de gestantes cadastradas. Optou-se por selecionar as Unidades que tinham o maior número de gestantes; somente nas regiões leste e oeste houve uma diferença na seleção, pois a primeira era campo de estágio da pesquisadora, facilitando assim o processo de coleta, e a segunda por questões de segurança pessoal da pesquisadora, já que nesta região a Unidade que possuía o maior número de gestantes cadastradas tinha um alto índice de violência.

Foram entrevistadas somente gestantes que aceitaram participar da pesquisa, assinando o Termo de Consentimento Livre e Esclarecido (TCLE), conforme Resolução 196/96 CNS. Para gestantes menores de 18 anos, foi solicitado o consentimento dos pais ou responsável. A justificativa para incluir as adolescentes está no fato de que a gravidez na adolescência é considerada um problema importante de saúde pública, influenciando os coeficientes tanto de mortalidade materna como infantil, assim como a incidência de prematuridade. Associado a isso, é na adolescência que, segundo pesquisadores do tema, se inicia o hábito do consumo de bebida alcoólica e drogas psicoativas, pelo próprio comportamento de busca do novo e atitudes de risco do adolescente.
Portanto, a exclusão de gestantes adolescentes seria uma perda significativa na qualidade dos dados da pesquisa, deixando de subsidiar os serviços de saúde sobre a importância da assistência adequada a essa população.

$\mathrm{Na}$ entrevista, foram utilizados dois instrumentos. O primeiro foi um formulário com as seguintes partes: dados sociodemográficos, histórico gestacional e saúde da gestante. Foi utilizado como critério para definição da classificação socioeconômica o instrumento de classificação da Associação Brasileira de Empresas e Pesquisas ${ }^{(12)}$.

O segundo instrumento utilizado foi o T-ACE que é um instrumento de rastreamento do uso de álcool. Desenvolvido por Sokol e Claren ${ }^{(6)}$, em 1989. Este instrumento foi validado para a língua portuguesa em 2002, por Fabbri ${ }^{(4)}$. Durante o estudo brasileiro de validação do T-ACE, os autores observaram o desconforto e resistência causados pela simples apresentação das questões. Sendo assim, a versão brasileira foi adaptada com o objetivo de melhorar a sua aceitação pelas gestantes ${ }^{(1)}$.

O objetivo de T-ACE é identificar casos de risco para o desenvolvimento da Síndrome Fetal do Álcool, avaliando a tolerância do álcool em gestantes. A sigla tem como significado "T-tolerance", "A- annoying", "C-cut down" e "E-eye opener", sendo que cada letra significa um quesito avaliado em cada questão, que são: letra T - nível de tolerância ao álcool; letra A - sentir-se agredido pela crítica dos outros; letra C - desejo de interromper o consumo; e letra E - necessidade de beber pela manhã para sentir-se melhor. Para pontuação, considera-se de zero a dois pontos para a primeira questão e um ponto para as questões subsequentes. A pontuação máxima é de cinco pontos e o limite para considerar um caso como positivo para o T-ACE são dois pontos ${ }^{(1)}$.

A primeira questão deste instrumento avalia a tolerância, considerando o consumo diário de 28 gramas de álcool absoluto, o que seria equivalente, por exemplo, a duas latas de cerveja. Portanto, a pontuação seria equivalente a menor ou igual a 2 na primeira questão, o que caracterizaria a gestante como um caso positivo para o T-ACE. As demais questões complementam o instrumento, somando pontos para finalizar o rastreamento. Casos positivos implicam em uso problemático do álcool e, como consequência, maior chance de desenvolvimento de problemas para o feto.

As gestantes foram abordadas, em sua maioria, 
no momento que compareceram às UBS para a realização da consulta pré-natal. Porém, foi necessária uma complementação da amostra, sendo então realizada pela pesquisadora a busca ativa das gestantes nos domicílios.

Os principais motivos de perdas de sujeitos da pesquisa foram: não-localização das gestantes, faltas às consultas pré-natais e nascimento dos bebês antes da entrevista.

Foram realizadas análises estatísticas da ocorrência do consumo de álcool pelas gestantes em Londrina, e a caracterização dos fatores que podem estar relacionados como, por exemplo, nível socioeconômico, idade, escolaridade, fatores ambientais (família, amigos), culturais, dentre outros. Os dados são apresentados em frequências absolutas e relativas.

O presente trabalho teve aprovação do Comitê de Ética em Pesquisa da Universidade Estadual de Londrina, sob protocolo n. 085/07.

\section{RESULTADOS}

O total de gestantes entrevistadas foi de 202 , sendo que $11,8 \%$ residiam na região central, $16,8 \%$ na região oeste, $17,3 \%$ na região leste, $25,2 \%$ na região sul e $28,7 \%$ na região norte da cidade.

A idade das 202 gestantes entrevistadas variou de 13 a 41 anos, sendo que a maioria delas $(66,8 \%)$ tinha idades entre 20 a 35 anos. Observou-se que $22,5 \%$ gestantes eram adolescentes, entre 12 e 19 anos. A maior parte $(40,6 \%)$ pertencia à classe média baixa, seguida pela classe pobre (30,7\%). E, dentre as entrevistadas, $87,6 \%$ relataram ter companheiro fixo, enquanto que $12,4 \%$ afirmaram estar solteiras.

Quanto à pontuação no T-ACE, $16,8 \%$ das gestantes fizeram pontuação mínima para classificação como risco, $2 \%$ atingiram mais de três pontos no rastreamento, e apenas uma $(0,5 \%)$ fez pontuação máxima (5 pontos), totalizando $19,3 \%$ das gestantes com T-ACE positivo. As demais (80,7\%) não pontuaram, ou fizeram apenas um ponto.

A região que apresentou maior número de gestantes que pontuaram para a maior classificação de risco foi a leste, onde foram entrevistadas 35 gestantes, das quais $48,5 \%$ fizeram, no mínimo, dois pontos. Deve ser considerado que nesta região a pesquisadora que realizava as entrevistas tinha maior afinidade com as gestantes, por ser seu campo de estágio, o que pode ter influenciado em maior fidedignidade das respostas, devido à formação prévia de um vínculo.

Entre as gestantes de 12 a 19 anos, $15 \%$ apresentaram T-ACE positivo, fazendo 2 pontos e 1 $(0,5 \%)$ fez 3 pontos, totalizando $6,4 \%$ das entrevistadas. Já entre as mulheres de 20 a 35 anos, 10,9\% apresentaram T-ACE positivo com 2 pontos e $1 \%$ com 3 pontos, correspondendo a $11,9 \%$ do total. Nas gestantes com idade maior que 35 anos, $1 \%$ classificou-se como risco, fazendo 2 pontos, e $0,5 \%$ fez pontuação máxima. Ressalta-se que aproximadamente $19 \%$ de todas as entrevistadas, independente da idade, foram classificadas como risco para o consumo de álcool durante a gestação.

Dentre as mulheres que classificaram-se como T-ACE positivo, com um mínimo de 2 pontos, 7,4\% pertencem à classe média baixa e 4,5\% à classe pobre, sendo que apenas $1,5 \%$ fazem parte da classe média alta, $3 \%$ da classe média e $0,5 \%$ da classe abaixo da linha da pobreza.

Dentre as 177 mulheres que afirmaram ter um companheiro, $13,4 \%$ classificaram-se como de risco no T-ACE com 2 pontos; $1,5 \%$ fizeram 3 pontos e $0,5 \%$ fez 5 pontos, totalizando $15,3 \%$. Já entre as 25 mulheres que afirmaram estar sozinhas, apenas 3,5\% fizeram 2 pontos, sendo que nenhuma fez pontuação maior que esta.

Quando questionadas sobre antecedentes familiares de problemas com álcool, 44,05\% das gestantes relataram ter algum parente próximo com problemas neste sentido, sendo que pai, avô e tios foram os mais citados $(86,5 \%)$, enquanto o companheiro conjugal apareceu em $13,4 \%$ das respostas.

Quando questionadas sobre a ocorrêndia de problemas devido ao consumo de álcool antes da gestação, como faltas ao trabalho, brigas, envolvimento policial entre outros, $8,9 \%$ das mulheres responderam afirmativamente, enquanto a maioria $(91,1 \%)$ negou qualquer tipo de problema em relação ao consumo de álcool. Dentre as que responderam "sim", os principais problemas citados foram as faltas ao trabalho e brigas entre familiares. E, quando questionadas sobre problemas devido ao uso de álcool durante a gestação, todas negaram qualquer tipo de problema, até mesmo as grandes pontuadoras no T-ACE.

\section{DISCUSSÃO}

Durante a realização das entrevistas, observou-se que a maioria das mulheres demonstrou desconforto em oferecer dados em relação ao seu consumo de álcool.

Entre as mulheres que foram classificadas 
como T-ACE positivo, com um mínimo de 2 pontos, verificou-se que a maioria pertencia às classes média baixa e à classe pobre. Diante disto, podemos evidenciar uma correlação entre nível econômico e consumo de álcool, pois foi entre as classes mais baixas que os índices de classificação do uso nocivo de álcool se apresentou expressivo. Embora a classe abaixo da linha da pobreza foi a que apresentou menor índice de pontuação, a classe pobre foi a que apresentou o maior número de pontuadoras, onde $4,5 \%$ fizeram dois pontos, $1,5 \%$ fizeram três pontos e $0,5 \%$ fez 5 pontos, reafirmando o que foi descrito acima.

Neste estudo, não houve associação entre solteiras e o consumo de álcool, já que os maiores índices de pontuação foram entre as mulheres que se declararam com companheiro fixo.

Na literatura, é relatada uma associação significativa entre consumo de bebida alcoólica e gestantes solteiras ${ }^{(13)}$. Alguns estudos demonstram uma ocorrência duas vezes maior de consumo de bebida alcoólica durante a gravidez entre mulheres solteiras ao compará-las com casadas, provavelmente porque a gestação em mulheres solteiras, em geral, está associada a outros fatores de risco para o consumo de bebida alcoólica, como baixa escolaridade, baixo nível socioeconômico e gravidez indesejada ${ }^{(14)}$, fato este que não foi encontrado neste estudo. Acredita-se que por se declararem estar com companheiro, esse consumo referido poderia ser em eventos sociais do casal e não tanto em locais frequentados por jovens solteiros.

A questão socioeconômica tem sido associada ao consumo de álcool ${ }^{(15)}$. Na população estudada, observou-se que a maioria das gestantes enquadravam-se na classe econômica média baixa, seguida logo após pela classe pobre. Entretanto, para melhor entendimento da associação entre alcoolismo e nível socioeconômico, seriam necessários estudos estruturados com este objetivo, não utilizando dados aproveitados de inquéritos, pois dados assim necessitam de um número suficiente de alcoolistas para constituir grupos adequados de comparação ${ }^{(16)}$.

Porém, em um estudo realizado com uma população de 2.177 indivíduos adultos ( 20 a 69 anos), demonstrou-se que a associação entre o consumo abusivo de álcool e o nível socioeconômico era inversa, ou seja, houve um aumento da prevalência do consumo de álcool de acordo com a diminuição da classe social. Este mesmo estudo demonstrou que, entre as mulheres, a faixa etária mais jovem foi a que apresentou maior tendência ao uso abusivo do álcool ${ }^{(16)}$.
Em outro estudo, encontrou-se como principal influência do alcoolismo as induções do ambiente familiar, ou seja, é geralmente no ambiente familiar que o sujeito tem seu primeiro contato com a bebida alcoólica, em geral por influência do pai ou parente próximo, e, normalmente, isto ocorre por volta dos 10 a 15 anos de idade ${ }^{(17)}$.

Ressalta-se que o uso nocivo de álcool deve ser entendido como um padrão de consumo que pode causar danos tanto físico quanto mental à saúde de um indivíduo, pois observa-se que, normalmente, o usuário enfrenta criticas de familiares e/ou de outras pessoas, associando-se ainda a vários tipos de consequências sociais.

O consumo dependente ou alcoolismo intenso caracteriza-se por uma média de consumo maior que 45 drinques, em um mês, ou mais de 5 drinques em uma mesma ocasião. O consumo de bebida alcoólica é classificado como: leve (1,2 doses/dia), moderado (2,2 doses/dia), pesado (3,5 doses/dia) e abusivo (5,4 doses/ dia) ${ }^{(18)}$

No caso das gestantes, outro agravante seria o de que é no primeiro trimestre da gestação que o feto está mais suscetível à má-formação, pois é nesta fase que ocorre o desenvolvimento de órgãos e sistemas vitais, tais como coração, olhos, membros, entre outros. O consumo moderado de etílicos, quando feito no primeiro trimestre, pode ocasionar alterações no Sistema Nervoso Central, diretamente sobre a formação do túbulo neural ${ }^{(19)}$. Portanto, é de extrema importância avaliar a quantidade de bebida etílica ingerida, já que, como dito anteriormente, apenas 28 gramas (duas latas de cerveja) de álcool são suficientes para desencadear processos de má formação fetal.

Ainda não há um consenso sobre a quantidade tolerável de ingesta de álcool para gestantes. Porém, estudos comprovam que 20 gramas de álcool são suficientes para provocar depressão respiratória e dos movimentos fetais, observados via ultrassonografia ${ }^{(20)}$. De acordo com o T-ACE, o consumo maior ou igual a 28 gramas de álcool já é classificado como de risco para o desenvolvimento de anomalias fetais.

Pesquisas sobre consumo de bebida alcoólica durante a gravidez também observaram que a confirmação do consumo de etílicos durante a gestação não é fácil de ser obtida, relacionando isto ao provável constrangimento da mulher em informar seu real consumo, ou ainda o despreparo do profissional para investigar ou valorizar queixas que poderiam estar 
ligadas ao hábito de beber ${ }^{(20)}$.

Isto novamente questiona a confiabilidade das respostas das gestantes e sugere a pergunta de quais seriam as estratégias e meios mais adequados para conseguir respostas com maior confiabilidade.

\section{CONCLUSÃo}

A importância deste estudo deu-se de forma a mensurar a situação do consumo de álcool entre gestantes de Londrina/PR, relacionando isto aos problemas que podem acometer o feto, incluindo a Síndrome Alcoólica Fetal.

A detecção precoce do consumo de álcool permite a realização de intervenções que visem tanto a prevenção quanto a terapêutica. No entanto, a prevenção ainda é a melhor forma de evitar anormalidades ao feto.

Mesmo sabendo a dose que pode ser caracterizada como nociva, é recomendável a não-ingesta de etílicos, considerando que não se pode determinar se uma mesma quantidade é prejudicial ou não para mulheres diferentes, ou seja, uma quantidade nociva para uma poderia não ser para outra.

A Enfermagem pode atuar de maneira eficiente ao encorajar e incentivar a gestante a interromper o consumo de álcool durante as consultas pré-natais, mesmo que para isto seja necessário utilizar-se de argumentos que choquem e causem impacto. Assim, será possível promover menor chance de efeitos teratogênicos ao feto e garantindo melhor qualidade de vida à gestante.

\section{REFERÊNCIAS}

1. Pinheiro SN. Consumo de álcool e problemas emocionais em gestantes em um serviço obstétrico na cidade de Ribeirão Preto-SP [dissertação]. Ribeirão Preto (SP): Universidade de São Paulo; 2003.

2. Madi PC. Alcoolismo feminino. 2000. [acesso em 12 abr 2007]. Disponível: http://tiny.cc/tx4hv

3. Stewart SH, Zeitlin SB. Anxiety sensitivity and alcohol use motives. J Anxiety Disord. 1995;9(3):229-40.

4. Fabbri CE. Desenvolvimento e validação de um instrumento para rastreamento do uso nocivo do álcool durante a gavidez (T-ACE) [dissertação]. Ribeirão Preto (SP): Universidade de São Paulo; 2002.

5. Chaudhuri JD. Alcohol and developing fetus: a review.
Med Sci Monit. 2000;6(5):1031-41.

6. Sokol RJ, Claren SK. Guidelines for use of terminology describing the impact of prenatal alcohol on the offspring. Alcohol Clin Exp Res. 1989;13(4):597-98.

7. Paineras LL. Narrativas sobre a estimulação precoce evidenciando as particularidades de crianças portadoras da Síndrome Alcoólica Fetal [dissertação]. Rio de Janeiro: Fundação Osvaldo Cruz; 2005.

8. Sampson PD, Streissguth AP, Bookstein FL, Little RE, Clarren SK, Dehaene P, et al. Incidence of fetal alcohol syndrome and prevalence of alcoholrelated neurodevelopmental disorder. Teratology. 1997;56(5):317-26.

9. National Center on Birth Defects and Developmental Disabilities. Centers for Disease Control and Prevention. Department of Health and Human Services. Fetal alcohol syndrome: guidelines for referral and diagnosis. 2004. [acesso em 02 abr 2010]. Disponível: http://tiny.cc/14731

10. Floyd RL, O'Connor MJ, Sokol RJ, Bertrand J, Cordero JF. Recognition and prevention of fetal alcohol syndrome. Obstetrics and Gynecology. 2005;106(5 Pt 1):1059-64.

11. Ministério da Saúde (BR). SISPRENATAL: sistema de pré-natal. [acesso em 20 maio 2010]. Disponível: http://tiny.cc/rhki7

12. Associação Brasileira de Empresas de Pesquisa. Critério de classificação econômica brasil. [acesso em 20 mai 2010]. Disponível: http://www.marketanalysis. com.br/arquivos-download/biblioteca/cceb-1.pdf

13. Burd L, Martsolf J, Klug MG, O'Connor E, Peterson M. Prenatal alcohol exposure assessment: multiple embedded measures in a prenatal questionnaire. Neurotoxicol Teratol. 2003;25(6):675-79.

14. Leonardson GR, Loudenburg R. Risk factors for alcohol use during pregnancy in a multistate area. Neurotoxicol Teratol. 2003;25(6):651-58.

15. Silva LVR, Malbergier A, Stempliuk VA, Andrade AG. Fatores associados ao consume de álcool e drogas entre estudantes universitários. Rev Saúde Publica. [Internet] 2006; 40(2) [acesso em 08 jul 2009]. Disponível: http://tiny.cc/ixs21

16. Costa JSD, Silveira MF, Gazalle FK, Oliveira SS, Hallai PC, Menezes AMB, et al. Consumo abusivo de álcool 
e fatores associados: estudo de base populacional. Rev Saúde Pública. [Internet] 2004;38(2) [acesso em 08 jul 2009]. Disponível: http://www.scielosp.org/pdf/rsp/ v38n2/19790.pdf

17. Nascimento EC, Justo JS. Vidas errantes e alcoolismo: uma questão social Universidade Estadual Paulista. Psicol Reflex Crit. 2000;13(3):529-38.

18. Moore KL, Persaud TVN. Embriologia clínica. Rio de Janeiro: Guanabara Koogan; 1994.

19. Eckardt MJ, File SE, Gessa GL, Grant KA. Effects of moderate alcohol consumption on the central nervous system. Alcohol Clin Esp Res. 1998;22(5):998-40.

20. Kaup ZOL, Merighi MAB, Tsunechiro MA. Avaliação do consumo de bebida alcoólica durante a gravidez. Rev Bras Ginecol Obstet. [Internet] 2001;23(9):57580 [acesso em 20 maio 2009]. Disponível: http://tiny. $\mathrm{cc} / 2 \mathrm{fg} 8 \mathrm{f}$ 